\title{
Numerical investigation on evolution of cylindrical cellular detonation *
}

\author{
WANG Chun (王春) ${ }^{1}$ ， JIANG Zong-lin (姜宗林) ${ }^{1}$, \\ HU Zong-min (胡宗民) $)^{1,2}$, HAN Gui-lai (韩桂来) ${ }^{1}$ \\ (1. Institute of Mechanics, Chinese Academy of Sciences, Beijing 100190, P. R. China; \\ 2. ReCAPT, Gyeongsang National University, Jinju, Kyeongnam 660-701, South Korea)
}

(Communicated by ZHOU Zhe-wei)

\begin{abstract}
Cylindrical cellular detonation is numerically investigated by solving twodimensional reactive Euler equations with a finite volume method on a two-dimensional self-adaptive unstructured mesh. The one-step reversible chemical reaction model is applied to simplify the control parameters of chemical reaction. Numerical results demonstrate the evolution of cellular cell splitting of cylindrical cellular detonation explored in experimentas. Split of cellular structures shows different features in the near-field and far-field from the initiation zone. Variation of the local curvature is a key factor in the behavior of cell split of cylindrical cellular detonation in propagation. Numerical results show that split of cellular structures comes from the self-organization of transverse waves corresponding to the development of small disturbances along the detonation front related to detonation instability.
\end{abstract}

Key words detonation, cellular instability, chemical reaction, shock, numerical simulation

Chinese Library Classification $\quad$ O381

2000 Mathematics Subject Classification 80 A25

\section{Introduction}

In the propagation of multi-dimensional detonation, cellular structures can be recorded on the smoke foil. Many experimental and numerical results have shown that the size of cellular structures is a characteristic parameter for the detonation of a certain detonable gas mixture ${ }^{[1]}$. Transverse waves play an important role for the formation and evolution of cellular structures of detonation, which results in the collision of triple-point structures along the detonation front. The regularity of the cellular structure depends on chemical kinetics of certain detonable gas mixtures. In the detonation of detonable gas mixtures diluted with inert gas, regular cellular structures can be observed in experiments. Numerical analysis to the detonation has illustrated irregular cellular structures with high active energy. There are some special cases for studying cellular evolution behaviors in the propagation of detonation. In the propagation of the cylindrical detonation, the total number of cellular structures cannot remain constant

* Received Apr. 11, 2008 / Revised Sept. 22, 2008

Project supported by the National Natural Science Foundation of China (No. 90205027) and China

Postdoctoral Science Foundation (No. 2005037444)

Corresponding author WANG Chun, Doctor, E-mail: wangchun@imech.ac.cn 
with the expansion of the front area of the detonation wave. That is, with the evolution of cellular structures, cellular cells splitting takes place to match the constant size of cellular cells, especially for the diverging cylindrical detonation with regular cells. The phenomenon is often termed as the cellular cell bifurcation. An experimental result conducted by Soloukhin in 1963 is illustrated in Fig. ${ }^{[2]}$. Lee et al. had discussed this. It showed that with the increase of the surface area of the diverging detonation, the formation of more than one localized explosion at the end of the cycle of a decaying blast wavelet was required ${ }^{[3]}$.

Current theories of detonation instability are mostly concerned with the instability of planar detonations under conditions of small disturbances. For example, with Fourier decomposition of the linearized reactive hydrodynamics equations, the detonation instability problem can be reduced to a one-dimensional, time-dependent problem given transverse wave number as $\varepsilon / 2 \pi^{[4]}$. The "square-wave" model of a detonation is also used to study the instability of one-dimensional detonation wave $e^{[5,6]}$. For diverging cylindrical or spherical detonations, the generation of new transverse waves is due to the cellular instability, that is, the self-organization of transverse waves in the detonation front.

In this paper, the instability of cylindrical cellular detonation is considered, and the mechanisms of the self-organized regeneration of transverse waves are investigated. Two-dimensional Euler equations implemented with one-step reversible chemical reaction model are solved by the finite volume method on a two-dimensional adaptive unstructured mesh.

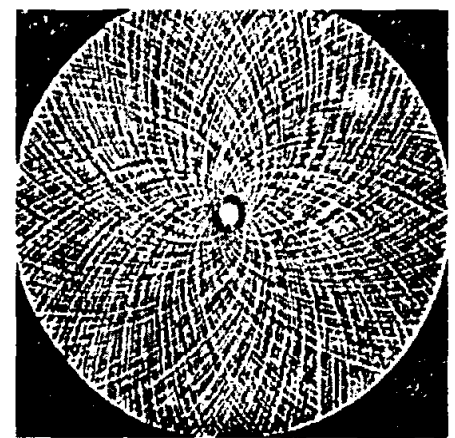

Fig. 1 Open-shuttle photograph of a diverging cylindrical detonation in a $\mathrm{C}_{2} \mathrm{H}_{2}-\mathrm{O}_{2}$ mixture

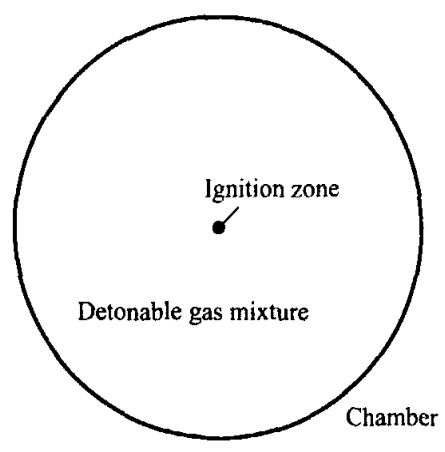

Fig. 2 The model for numerical simulation

\section{Problem specification}

As shown in Fig. 2, a certain detonable gas mixture is first charged in a cylindrical chamber. At the center of the chamber, a strong ignition source directly initiates detonation in the detonable gas mixture.

If the ignition source is strong enough, an over-driven detonation will be generated at the beginning. After a short time, the over-driven detonation decays fast to a cylindrical detonation. In the propagation of the cylindrical detonation, small disturbances will develop to strong disturbances, which results in the formation of transverse waves in the cylindrical detonation front under certain conditions. With the propagation of transverse waves and collision of the triple-point, the cellular cell can be recorded on the soot foil.

\section{Governing equations and numerical method}

When the behaviors of the detonation front are studied, the effects of viscosity, heat conductivity, diffusion of species and temperature can be neglected. With the above hypothesis, the 
cylindrically diverging detonation is governed by a two-dimensional Euler equation. For convenient analysis of the influence of chemical kinetics, the one-step reversible chemical reaction model is used in this paper. The governing equations in Cartesian coordinates can be expressed as

$$
\frac{\partial \boldsymbol{U}}{\partial t}+\frac{\partial \boldsymbol{F}}{\partial x}+\frac{\partial \boldsymbol{G}}{\partial y}=\boldsymbol{S}
$$

where

$$
\begin{aligned}
\boldsymbol{U} & =[\rho, \rho u, \rho v, \rho E, \rho \lambda]^{\mathrm{T}}, \\
\boldsymbol{F} & =\left[\rho u, \rho u^{2}+p, \rho u v,(\rho E+p) u, \rho \lambda u\right]^{\mathrm{T}}, \\
\boldsymbol{G} & =\left[\rho v, \rho u v, \rho v^{2}+p,(\rho E+p) v, \rho \lambda v\right]^{\mathrm{T}}, \\
\boldsymbol{S} & =[0,0,0,0, W]^{\mathrm{T}} .
\end{aligned}
$$

In the above expressions, $\rho, p$, and $E$ are the density, pressure and specific total energy of the fluid, respectively; $u$ and $v$ are the fluid velocities in the $x$ direction and the $y$ direction; $\lambda$ represents the progress variable measuring chemical reaction and $W$ is the chemical reaction source term. The total encrgy $E$ can be expressed as:

$$
E=\frac{p}{(\gamma-1) \rho}+\frac{1}{2}\left(u^{2}+v^{2}\right)-\lambda Q
$$

where $\gamma$ is the polytropic exponent of the gas mixture, and $Q$ is the chemical heat per unit mass.

For the one-step reversible chemical reaction with constant total mole number, the chemical reaction source can be expressed by a simplified Arrhenius law:

$$
W=\left[k_{\mathrm{f}}(1-\lambda)-k_{\mathrm{r}} \lambda\right] \mathrm{e}^{-E_{\mathrm{a}} / R_{\mathrm{o}} T},
$$

where $k_{\mathrm{f}}$ and $k_{\mathrm{r}}$ represent the forward and reverse reaction rates, respectively; $E_{\mathrm{a}}, R_{\mathrm{o}}$, and $T$ are the active energy, general gas constant, and temperature. $T$ is given as

$$
T=p / \rho R
$$

where $R$ is the gas constant of the reactant and product. For the reversible chemical reaction, $k_{\mathrm{r}}$ is related to $k_{\mathrm{f}}$ through the equilibrium constant $\pi_{\mathrm{e}}$ by

$$
k_{\mathrm{r}}=k_{\mathrm{f}} / \pi_{\mathbf{e}}
$$

Without considering the effects of viscosity, heat conductivity and diffusion, the reactive flow is determined by the constant parameters $k_{\mathrm{f}}, \pi_{\mathrm{e}}, E_{\mathrm{a}}, Q, \gamma$ and $R$, as well as the initial state parameters $p_{0}$ and $T_{0}$.

The governing equations can be transformed to the following expression on the control volume based on the finite volume method,

$$
\iint_{\Omega} \frac{\partial \boldsymbol{U}}{\partial t} d x d y+\int_{\Gamma}(\boldsymbol{F} d y+\boldsymbol{G} d x)=\iint_{\Omega} \boldsymbol{S} d x d y
$$

where $\Omega$ is the control volume and $\Gamma$ is the boundary of the control volume. As shown in Fig. 3 , where $U_{\mathrm{L}}$ and $\boldsymbol{U}_{\mathrm{R}}$ are the conservative variable on the left and right sides of the

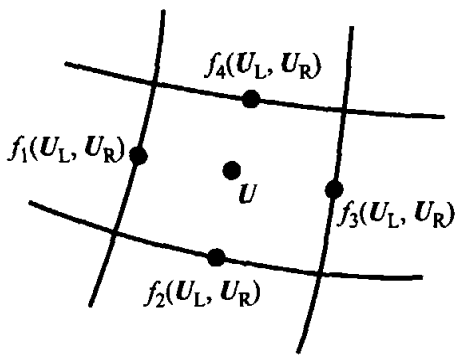

Fig. 3 The control cell for numerical simulation 
boundary of control volume. The unstructured meshes are the quadrangular cell; the variables are fixed on the center of the control volume; and the fluxes are counted through the boundary of the control volume. The fluxes on the boundary of control cells are constructed by HLLC algorithms. And the reconstructions of the variables on the center of control cells are computed by the 2nd order MUSCL algorithm construction and 1st-order temporal integration ${ }^{[7-8]}$.

The unstructured mesh can be refined adaptively according to the density gradient up to two layers and the minimal grid size up to $\Delta=0.02 \mathrm{~mm}$. The valve parameter for the mesh refinement is defined as

$$
\varepsilon_{\mathrm{T}}=\operatorname{Max}\left[\frac{\left(\nabla_{l} \rho\right)_{\mathrm{c}}-\left(\nabla_{l} \rho\right)_{i} \mid}{\alpha_{\mathrm{f}} \rho_{\mathrm{c}} / d l+\left|\left(\nabla_{l} \rho\right)_{i}\right|}, \frac{\left(\nabla_{l} \rho\right)_{\mathrm{c}}-\left(\nabla_{l} \rho\right)_{j} \mid}{\alpha_{\mathrm{f}} \rho_{\mathrm{c}} / d l+\left|\left(\nabla_{l} \rho\right)_{i}\right|}\right]
$$

where $i$ and $j$ denote the two adjacent control volumes; c denotes the center position of the boundary between the two adjacent control volumes; $V_{l}$ and $d l$ represent the gradient and length from the center of volume $i$ to the center of volume $j ; \alpha_{\mathrm{f}}$ is a predefined factor to prevent the numerical singularity.

In our numerical simulations, the initial temperature and pressure of the detonable gas are specified at $300 \mathrm{~K}$ and $0.1013 \mathrm{MPa}$. The gas properties of detonable gas and detonation product are selected to be $R \equiv 287.096 \mathrm{~J} /(\mathrm{mol} \cdot \mathrm{K})$. The polytropic exponent is taken as $\gamma=1.4$. The control parameters of the chemical reaction are selected as: $k_{\mathrm{f}}=8.5 \times 10^{8}, \pi_{\mathrm{e}}=20$, $E_{\mathrm{a}}=1.6 \times 10^{5} \mathrm{~J}, Q=1.58 \times 10^{6} \mathrm{~J}$.

The radius of the chamber is $0.1 \mathrm{~m}$. To save computational time, $1 / 4$ geometry is computed, It should be noticed that in the center of the cylindrical camber, a small circular region is cut to avoid possible numerical oddness. The inner and outer boundaries are defined as free-slip solid walls, and the radial boundaries are defined as symmetrical boundaries. The initial ignition source is numerically given as a small region of high pressure and high temperature.

\section{Results and discussion}

\subsection{Cellular structure regularity of the cylindrical detonation}

For the one-step chemical reaction model, there are four key non-dimensional parameters for the behaviors on the detonation instability, polytropic exponent $\gamma$, chemical heat $\beta=$ $\gamma Q /\left(R_{\mathrm{o}} T_{\mathrm{s}}\right)$, activation energy $\theta=\gamma E_{\mathrm{a}} /\left(R_{\mathrm{o}} T_{\mathrm{s}}\right)$ and the detonation over drive $f=\left(D / D_{\mathrm{CJ}}\right)^{2}$, where $T_{\mathrm{s}}$ is the shock temperature, and $D_{\mathrm{CJ}}$ is the Mach number of C.J detonation which can be calculated in exact Rankine-Hugoniot conditions which handles the detonation as a discontinuity. Numerical results show that regular oscillation in one-dimensional detonation and regular cellular cells in the two-dimensional cellular detonation can be observed for the low activation energy, and there is bifurcation or instable oscillation in one-dimensional and irregular cellular cells in the two-dimensional cellular detonation observed for the large activation energy. Under numerical conditions of this paper, the non-dimensional activation energy is about $\theta=10.2$, which corresponds to the lower activation energy. The cellular cell of detonation in numerical simulation is in the range of regular detonation.

Commonly, the length of the reaction zone in detonation is an important parameter for the analysis to the dynamic parameter of detonation wave. For one-step chemical reaction model, the length of the reaction zone is an infinite length, so the half-reaction length is always defined as the characteristic length for chemical reaction which is defined as the distance from the shock to the point where half of the reactant is consumed, $l_{1 / 2}$. In numerical simulation, there is a lowest demand for the grid resolution in the half-reaction length in cellular detonation ${ }^{[9]}$, otherwise, the numerical dissipation will suppress the development of transverse waves in the detonation front. Figure 4(a) illustrates the definition of the half-reaction length and Fig. 4(b) gives the variation of half-reaction length via the Mach number of leading shock with the chemical reaction model. In our numerical simulation, there are about $10 \sim 15$ grid points in the 
half-reaction length, and initial disturbances are not needed to trigger the initial transverse waves. The development of transverse waves in the detonation comes from the detonation instability responding to small disturbances. In the propagation of cylindrical cellular detonation, the cellular structure is not attenuated by the numerical dissipation.

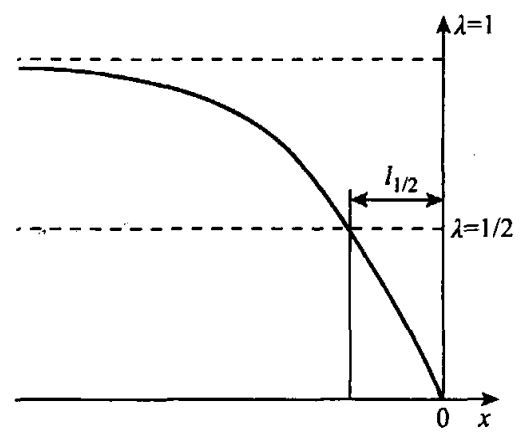

(a) The definition of $l_{1 / 2}$ in the shock frame

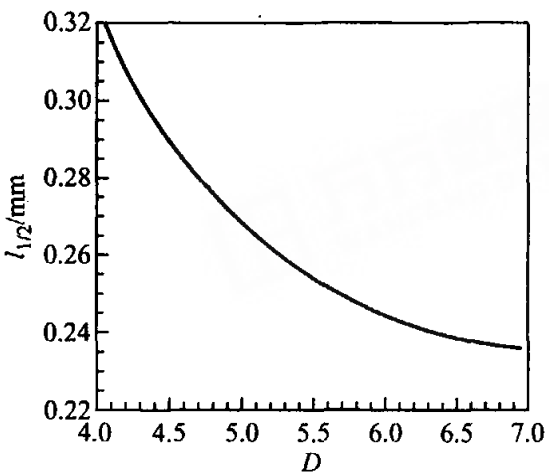

(b) $l_{1 / 2}(\mathrm{~mm})$ vs. detonation Mach number

Fig. 4 The definition of $l_{1 / 2}$ and its variation with the change in detonation Mach number

Figure 5 shows the numerical result of cellular structure evolution of the cylindrically diverging detonation. The cellular structures are recorded by the local maximum pressure, and the boundary of cells represents the loci of the tri-point structures in the detonation front. With low activation energy, numerical cells show quit regular. There are two regions in which cell evolution shows some different features. In the center region of the chamber, that is, the detonation initiation region, cellular cells develop very quickly. In the far-field of the cylindrical detonation, the generation of new cellular cells is comparatively slow. Figure 6 illustrates cellular structures of two typical region in the near-field and far-field. In the near-field of cellular cylindrical detonation, large number of new tri-point loci developes quickly between the original tri-point loci. In the far-field of cellular cylindrical detonation, the number of new tri-point loci is comparatively less than that in the near-field. Obviously, the variation of the curvature of the detonation front plays a key role for different features of the cellular structure evolution in the near-field and that in the far-field. The curvature variation of cylindrical detonation in the near-field is much larger than that in the far-field, which results in the fast generation of the new tri-point loci.

From Fig. 5 and Fig. 6, we can conclude that the regular-

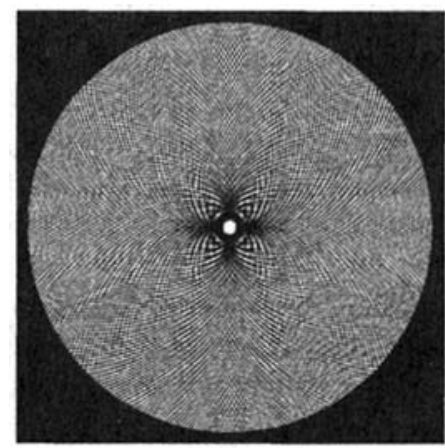

Fig. 5 Numerical result for cylindrical cellular detonation ity of cellular structures in the macro scale does mean that the generation of the new tri-point is regular in the micro scale. The generation of new tri-point structures greatly relies on local characteristics of detonation, such as the local curvature of the detonation front, gas dynamic properties and local chemical reaction.

\subsection{The self-organization of transverse waves in cylindrical detonation propagation}

The evolution of the cylindrical cellular detonation indicates a periodic process, in which cell splitting takes place when cells become sufficiently large with the area expansion of detonation front, that is, new transverse waves are generated by self-organization evolution to match the demand that cellular cells maintain a roughly similar size for a given gas mixture with given initial thermodynamic states. 


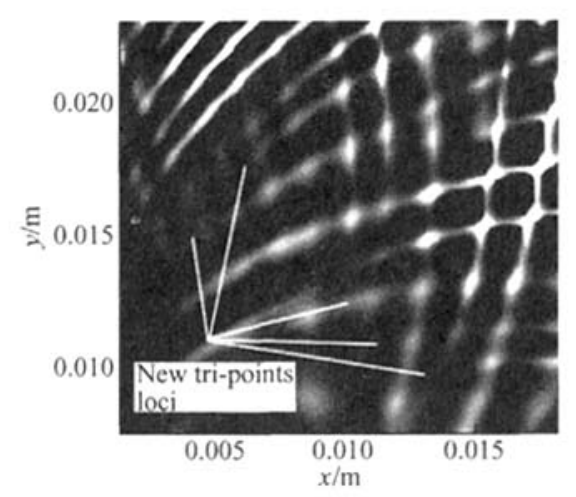

(a) Cells evolution in the nearfield

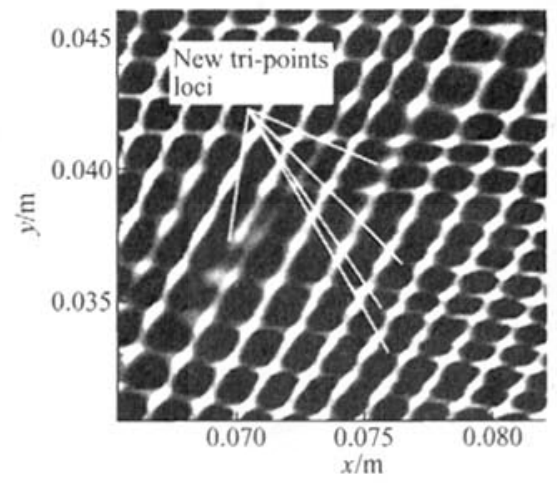

(b) Cells evolution in the farfield

Fig. 6 The local features of cellular cells evolution of cylindrical detonation

The modes of cell splitting in the diverging cylindrical detonation have been studied and summarized as the following modes, the over-expansion of detonation, wrinkled and kinked detonation front which greatly depends on the local characteristics of detonation mentioned before $^{[10-12]}$.

The mechanism of cellular splitting in cylindrical detonation propagation comes from the instability of detonation. For the multi-dimensional detonation, the instability of detonation exhibits the instability of cellular cells in detonation. Furthermore, in the cylindrical cellular detonation, how the small disturbances propagating along the detonation front develop to the strong transverse wave is the main mechanism for the cell splitting in the expanded detonation.

Figure 7 shows 3-D view density distributions of four successive instants in the near-field. In the early stage of the cylindrical cellular detonation, the disturbed waves propagating along the detonation front develop to strong transverse waves in a short distance. By collisions of tripoint structures, old and new transverse waves adjust themselves to the proper interval along the detonation front. Obviously, the local expansion of cylindrical detonation is much faster than that of a planar detonation, especially in the near-field of detonation, which results in the fast splitting of cellular cells in cylindrical detonation propagation. There are several new transverse waves that are generated almost simultaneously.

Figure 8 shows the 3-D view density distributions of four successive instants in the farfield. In the far-field of the cylindrical cellular detonation, the local expansion of cylindrical detonation is slow, and the disturbed waves propagating along the detonation front develop to strong transverse waves in a long distance. By the collisions of tri-point structures, old and new transverse waves adjust themselves to the proper interval along the detonation front. Obviously, the local expansion of cylindrical detonation in the far-field is much slower than that in the near-field, which results in the slower splitting of cellular cclls in the far-field.

Figure 9 shows the shock pressure distribution versus the radius along the line of a 45-degree angle. In the initial stage, the strong detonation is initiated and then decays quickly. The decayed detonation undergoes a stable stage, which corresponds to regular cellular structures. From the position at about $r=0.02 \mathrm{~m}$, the cells incline to irregular cells, which shows that irregular shock pressure oscillations appear and new transverse waves are generated. After the self-organization of transverse waves, at the position of about $r=0.04 \mathrm{~m}$, there is a stage of regular cells.

Form the position of about $r=0.07 \mathrm{~m}$, cellular cells prepare for the next splitting, which shows irregular shock pressure oscillations. These show that the cellular cell splitting takes place stage by stage. There are certain periods for stable cells and unstable cells in the propagation 

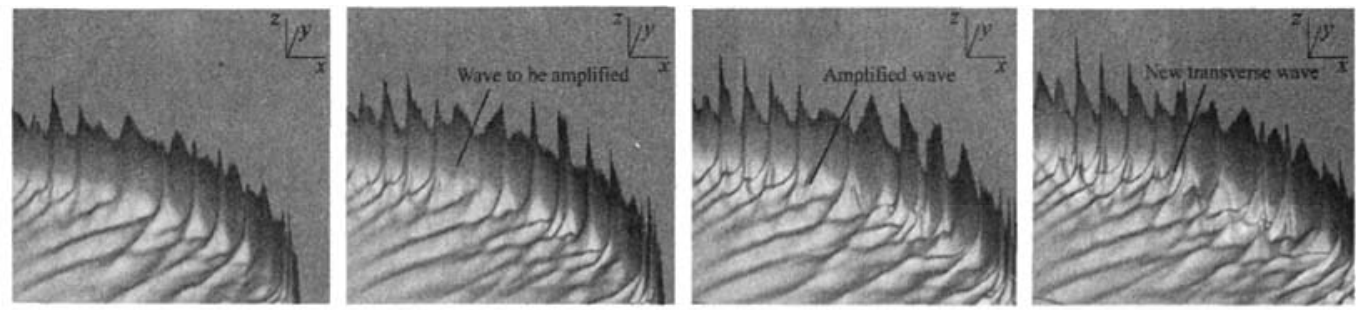

Fig. 7 3-D view density distributions of four successive instants in the near-field
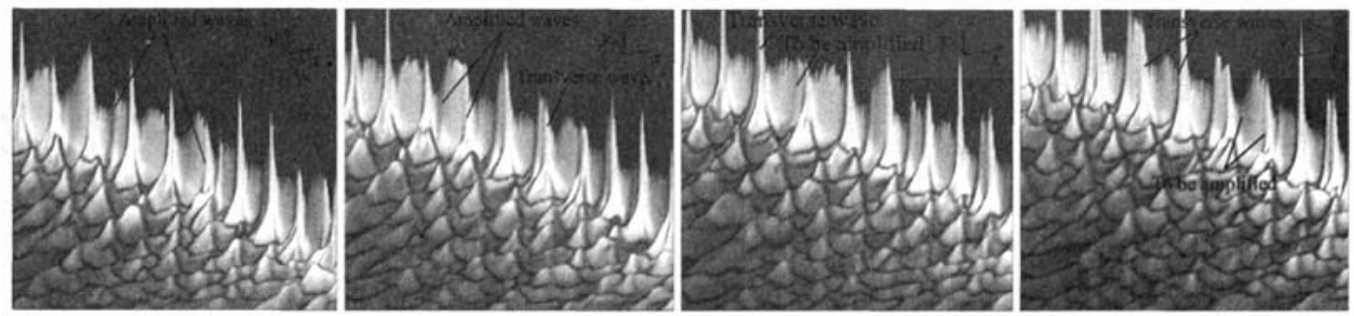

Fig. 8 3-D view density distributions of four successive instants in the far-field

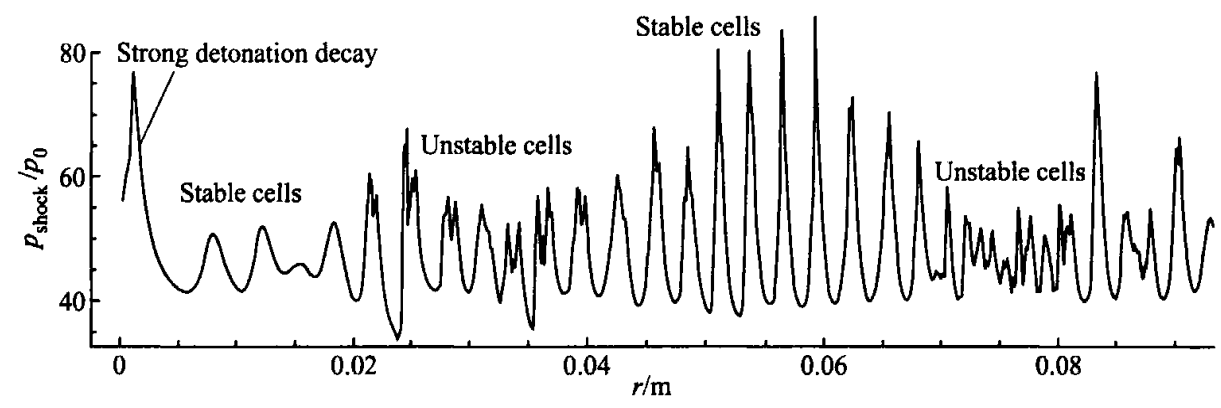

Fig. 9 The shock pressure vs. radius of cylindrical detonation propagation

of cylindrical cellular detonation, shock pressure oscillations reflect such shift mechanism of cell development, which comes from the self-organization of transverse waves in the detonation front.

The self-organized regeneration of transverse waves in this paper is a typical characteristic of cylindrically diverging detonations, which reflects the basic mechanism of detonation instability in a different manner from planar detonation.

\subsection{The travelling of the transverse waves along the detonation front}

It has been accepted that the size of cellular cells is a characteristic size for the detonation of certain gas mixture given certain initial conditions, which is used to build some relations -to dynamic parameters of a detonation, such as initiation energy, critical diameter, etc. The propagation of cylindrical cellular detonation inclines the length of detonation front to integral times of the cell size, especially for a cylindrical detonation with regular cellular cells. From Fig. 1 to Fig. 5, we can find that the traveling of the tri-point structures on the cylindrical detonation front is along a spiral locus around the detonation initiation region. All spirals are similar, which means the spiral loci may be a characteristic phenomena of a cylindrical cellular detonation. 
The spiral loci of tri-point structures may be defined as:

$$
V_{r}=D, \quad V_{\theta}=W_{\mathrm{d}} / r
$$

where $D$ is the detonation speed and $W_{\mathrm{d}}$ is the speed of transverse waves propagating along the detonation front. In the near-field of cylindrical detonation, $D$ and $W_{\mathrm{d}}$ greatly depend on the detonation over drive $f$. If the radius of detonation propagation inclines to infinity, $V_{\theta}$ will incline to zero, which means the cylindrical detonation inclines to a planar detonation.

\section{Concluding remarks}

The cellular structure evolution of cylindrical detonation is numerically investigated with one-step chemical reaction model of low activation energy. It shows that the cell splitting in near-field is more influenced by the variation of curvature of cylindrical detonation front than that in far-field of cylindrical cellular detonation. The variation of shock pressure exhibits the strong detonation decay and pcriodic behaviors of stable cell and unstable cell in the process of cell splitting in the propagation of cylindrical cellular detonation. The generation of new transverse waves comes from the development of small disturbances along the detonation front related to cylindrical detonation instability. The equation of spiral loci of tri-point structures is mathematically established, which shows distinct difference from the planar cellular detonation.

Acknowledgements The authors would like to thank $\mathrm{Dr} \mathrm{Li} \mathrm{H} \mathrm{H}$ for providing the codes.

\section{References}

[1] Lee J H S, Lee B. Cylindrical imploding shock waves[J]. Physics of Fluids, 1965, 8(12):2148 2152.

[2] Sun C W, Wei Y Z, Zhou Z K. Applied detonation physics [M]. Beijing: Defense Industry Press, 2000, 3: p90-94 (in Chinese).

[3] Lee J H S. Initiation of gaseous detonation[J]. Ann Rev Phys Chem, 1977, 28(3):75-104.

[4] Erpenbeck J J. Detonation stability for disturbance of small transverse wavelength[J]. Physics of Fluids, 1966, 9:1293-1306.

[5] Zaidel R M, Zeldovich Y B. One-dimensional instability and attenuation of detonation[J]. Zh Prikl Mekh i Tekh Fiz, 1963, (6):59-65.

[6] Alpert R L, Toong T Y. Periodicity in exothermic hypersonic flows about blunt projectiles[J]. Astronaut Acta, 1962, 17:539-560.

[7] Sun M. Numerical and experimental studies of shock wave interaction with bodies[D]. Ph D dissertation. Tohoku University, Japan, 1998.

[8] Li H H. Experimental and numerical study on unsteady complex flow and wave interaction[D]. $\mathrm{Ph}$ D dissertation. University of Science \& Technology of China, China, 2005.

[9] Christiane Helzel, Randall $\mathrm{J}$ Leveque, Gerald Warnecke. A modified fractional step method for the accurate approximation of detonation waves[J]. Siam J Sci Comput, 2000, 22(4):1489-1510.

[10] Watt S D, Sharpe G J. Linear and nonlinear dynamics of cylindrically and spherically expanding detonation waves[J]. Journal of Fluid Mechanics, 2005, 522(1):329-356.

[11] Wang C, Jiang Z L. Numerical simulation of the cellular structure cvolution in cylindrically diverging detonation[C]. In: Hannemann Klaus, Seiler Friedrich (eds). Proceeding of 26 th International Symposium on Shock Waves, Gottingen, Germany: Springer-Verlag, July 2007.

[12] Han G L, Jiang Z L, Wang C, Zhang F. Cellular cell bifurcation of cylindricul detonations[J]. Chinese Physics Letters, 2008, 25(6):2125-2127. 